\title{
Transmodal Sensorimotor Networks during Action Observation in Professional Pianists
}

\author{
B. Haslinger ${ }^{1}$, P. Erhard ${ }^{1}$, E. Altenmüller ${ }^{2}$, U. Schroeder ${ }^{1}$, \\ H. Boecker ${ }^{1}$, and A. O. Ceballos-Baumann ${ }^{1}$
}

\begin{abstract}
Audiovisual perception and imitation are essential for musical learning and skill acquisition. We compared professional pianists to musically naive controls with fMRI while observing piano playing finger-hand movements and serial finger-thumb opposition movements both with and without synchronous piano sound. Pianists showed stronger activations within a fronto-parieto-temporal network while observing piano playing compared to controls and contrasted to perception of serial finger-thumb opposition movements. Observation of silent piano playing additionally recruited
\end{abstract}

\section{INTRODUCTION}

Pianists spend years of extended and sustained training beginning at the earliest possible age in childhood in order to reach professional standard of musicianship comprising extreme upper-limb motor coordination linked to auditory and sensorimotor feedback. In this regard, musical skill acquisition shares many common elements with the development of speech. In analogy to infant learning of the native language, learning by listening, observation, and imitation of a teacher's actions are crucial steps in musical skill acquisition that are widely applied in music pedagogics.

Musical training involves a strong functional association between motor performance and somatosensory and auditory feedback. Professional musical training has been demonstrated to induce adaptive functional and structural changes within the motor system (Sluming et al., 2002; Amunts, Schlaug, et al., 1997; Elbert, Pantev, Wienbruch, Rockstroh, \& Taub, 1995; Schlaug, Jancke, Huang, Staiger, \& Steinmetz, 1995) as well as within the auditory system (Schneider et al., 2002; Pantev et al., 1998; Zatorre, Perry, Beckett, Westbury, \& Evans, 1998; Schlaug, Jancke, Huang, \& Steinmetz, 1995). Sergent, Zuck, Terriah, and MacDonald (1992) applied PET to study functional activations in musicians during musical sight-reading, active keyboard performance, and lis-

\footnotetext{
${ }^{1}$ TU München, Munich, Germany, ${ }^{2}$ Institut für Musikphysiologie und Musiker-Medizin, Hochschule für Musik und Theater, Hannover, Germany
}

auditory areas in pianists. Perception of piano sounds coupled with serial finger-thumb opposition movements evoked increased activation within the sensorimotor network. This indicates specialization of multimodal auditorysensorimotor systems within a fronto-parieto-temporal network by professional musical training. Musical "language," which is acquired by observation and imitation, seems to be tightly coupled to this network in accord with an observationexecution system linking visual and auditory perception to motor performance. tening to the corresponding tones. They demonstrated a functional association for these three domains within the premotor, inferior frontal (BA 44), and parietal areas. An EEG study suggested transmodal activation of the sensorimotor system by auditory input in professional musicians (Bangert, Haeusler, \& Altenmuller, 2001). Functional coupling of auditory and sensorimotor domains in musicians during passive observation of musical motor activity in association with corresponding acoustic input has not been studied with functional imaging so far.

Previous functional imaging and neurophysiological studies demonstrated involvement of a fronto-parietotemporal network during motor observation (Buccino et al., 2001; Grezes \& Decety, 2001; Perani et al., 2001; Nishitani \& Hari, 2000; Iacoboni, Woods, et al., 1999; Hari et al., 1998; Bonda, Petrides, Ostry, \& Evans, 1996; Grafton, Arbib, Fadiga, \& Rizzolatti, 1996; Rizzolatti, Fadiga, Matelli et al., 1996; Fadiga, Fogassi, Pavesi, \& Rizzolatti, 1995; Decety, Pereni, et al., 1994). They especially showed recruitment of inferior frontal areas including parts of the inferior frontal gyrus (BA 44, 45) and of the ventral premotor cortex (vPMC), of inferior parietal/intraparietal areas as well as of the superior temporal cortex (superior temporal sulcus [STS]) during motor observation. Furthermore, activations within dorsal parts of the lateral premotor cortex (dPMC) as well as within mesial premotor, prefrontal, and superior parietal cortices were associated with passive observation of movements. 
Our fMRI study comparing professional pianists to musically naive controls aimed at investigating the influence of musical training upon specialization of the cortical motor observation network for musical activity and at studying transmodal auditory and sensorimotor activations. Observation of piano-related finger movements was compared to perception of serial fingerthumb opposition movements both with and without piano sound. We expected stronger activations within the fronto-parieto-temporal network in musicians during observation of meaningful piano-related movements compared to observation of a resting hand and of serial finger-thumb opposition movements. Furthermore, we hypothesized a transmodal recruitment of auditory association areas and of sensorimotor areas in pianists by perception of piano playing movements or piano tones, respectively.

\section{RESULTS}

\section{Differential Activation during Observation of Meaningful Piano Playing Movements}

We determined activation differences between musicians and musically naive controls when comparing observation of piano playing movements without sound to observation of a resting hand. This contrast was performed separately for right- and left-hand movements. For observation of right-hand piano-related movements, professional musicians showed significantly greater activation than controls within a cortical frontoparieto-temporal network: in the inferior frontal cortex including the bilateral ventral parts of BA 6 (vPMC) and the opercular part of the inferior frontal gyrus (BA 44), as well as the bilateral inferior frontal gyrus, pars triangularis (BA 45), in the inferior parietal cortex (BA 40) (supramarginal gyrus) and the intraparietal sulcus (IPS) bilaterally and in the bilateral STS. Additionally, the superior frontal cortex/dPMC (BA 6), mesial premotor cortex/pre-SMA (BA 6), prefrontal cortex (BA 9/46), orbito-frontal cortex (BA 47), postcentral gyrus (BA 2), and superior parietal lobe (BA 7) were bilaterally stronger activated in musicians. Interestingly, observation of silent musical motor activity evoked stronger signal increases in pianists within the left primary auditory cortex/transverse temporal gyrus (BA 41) and the left superior temporal gyrus (BA 22, BA 42). Furthermore, musicians showed higher BOLD signal increases in the bilateral temporo-occipital cortex (BA 18/19/37), on the subcortical level in the cerebellar hemispheres and striatum bilaterally (Table 1, Figure 1).

For the comparison of observation of left-hand piano playing movements without sound versus observation of a resting hand between pianists and controls, a quite similar activation pattern was found. Differing from the results for right-hand movements, at the applied threshold, no activations were seen in the primary auditory cortex and within the bilateral superior parietal cortex (Table 1).

To examine whether piano sound in association with piano-related finger movements influences the activation differences, observation of piano playing movements with sound versus observation of a resting hand was contrasted between both groups. Professional musicians showed significantly greater activation than controls during observation of right-hand piano playing movements with piano sound in the left precentral cortex, vPMC (BA 6) at the border to BA 44, inferior parietal cortex (BA 40) (supramarginal gyrus) including a cluster within IPS bilaterally, left superior frontal cortex/dPMC (BA 6), left prefrontal cortex (BA 9), left postcentral cortex (BA 2), bilateral superior parietal cortex/precuneus (BA 7), right occipital cortex (BA 19), and both cerebellar hemispheres (Table 2).

Intergroup comparison for observation of left-hand piano playing with sound versus observation of a resting hand additionally showed higher activation in pianists in the left inferior frontal gyrus (BA 44, 45), right inferior frontal gyrus (BA 45), and right dPMC with no differences in prefrontal, intraparietal, superior parietal, occipital, and cerebellar areas (Table 2). Activation differences between both groups for observation of piano playing movements with sound versus observation of a resting hand for both hands were generally less extensive compared to the analysis of piano-related finger movements without sound. Here no activation differences between both groups were detected within secondary auditory areas.

\section{Differential Activation during Observation of Piano Playing Compared to Serial Finger-Thumb Opposition Movements}

Comparing observation of meaningful piano playing to observation of serial finger-thumb opposition movements without sound revealed activation differences between pianists and controls in a subset of the areas mentioned above suggesting their special role for the training dependent meaning of the observed action: for right-hand movements, the left inferior frontal cortex, namely, the vPMC (BA 6), opercular (BA 44) and prefrontal (BA 9) areas, right inferior parietal cortex (BA 40), IPS bilaterally, bilateral dorsal premotor cortex/dPMC (BA 6), postcentral cortex (BA 2/3) bilaterally, left parietal operculum (BA 43), right caudate nucleus, and bilateral cerebellar hemispheres were activated stronger in musicians (Table 3, Figure 2).

Activation changes in the intergroup comparison for left-hand movements differed with respect to vPMC activation that was stronger for musicians in the right hemisphere. No frontal opercular, prefrontal, intraparietal, and caudate signal differences were seen. Additionally, higher signal levels were detected for musicians 
Table 1. Fronto-Parieto-Temporal Areas with Stronger Activations in Musicians than in Controls during Observation of Piano Playing without Sound Versus Observation of a Resting Hand

\begin{tabular}{|c|c|c|c|c|c|c|c|c|c|c|c|c|}
\hline \multirow{2}{*}{$\frac{\text { Without Sound }}{\text { Area }}$} & \multicolumn{6}{|c|}{$R$ Piano $>$ Rest } & \multicolumn{6}{|c|}{ L Piano $>$ Rest } \\
\hline & Cluster & $B A$ & $x$ & $y$ & $z$ & T level & Cluster & $B A$ & $x$ & $y$ & $z$ & T level \\
\hline L sup. frontal, $d P M C$ & 1 & 6 & -20 & 1 & 57 & $6.24 *$ & 1 & 6 & -22 & 1 & 55 & $7.49 *$ \\
\hline Mesial premotor, pre-SMA & & 6 & -6 & 10 & 51 & $4.97 *$ & & 6 & -2 & 11 & 57 & $6.49 *$ \\
\hline $\mathrm{R}$ sup. frontal, $d P M C$ & & 6 & 18 & 7 & 53 & $5.04 *$ & & 6 & 20 & 8 & 53 & $6.30 *$ \\
\hline L mid. frontal, $v P M C$ & & 6 & -50 & 3 & 29 & $4.17 *$ & 2 & 6 & -51 & 4 & 31 & $5.49 *$ \\
\hline $\mathrm{L}$ inf. frontal, pars opercularis & & 44 & -50 & 10 & 20 & $3.76^{*}$ & 3 & 44 & -57 & 8 & 11 & $3.77 *$ \\
\hline L inf. frontal, pars triangularis & & $45^{\dagger}$ & -55 & 16 & 3 & $2.91 *$ & & 45 & -55 & 16 & 5 & $5.14^{*}$ \\
\hline $\mathrm{R}$ inf. frontal & 2 & 46 & 55 & 32 & 9 & $5.36 *$ & 4 & 46 & 55 & 28 & 10 & $3.57^{*}$ \\
\hline $\mathrm{R}$ inf. frontal, pars triangularis & & 45 & 51 & 29 & 2 & $4.07 *$ & & 45 & 53 & 26 & 8 & $4.17 *$ \\
\hline $\mathrm{R}$ inf. frontal, pars opercularis & & $44^{\dagger}$ & 55 & 12 & 3 & $2.90 *$ & & 44 & 55 & 12 & 3 & $4.08^{*}$ \\
\hline $\mathrm{R}$ mid. frontal, $v P M C$ & 3 & 6 & 42 & 2 & 48 & $4.69 *$ & 5 & 6 & 59 & 2 & 37 & $4.64 *$ \\
\hline $\mathrm{R}$ mid. frontal & 4 & 9 & 32 & 34 & 22 & $5.33 *$ & 6 & 46 & 50 & 46 & 18 & $3.63 *$ \\
\hline L mid. frontal & 5 & 46 & -42 & 40 & 26 & $4.17^{*}$ & 7 & $46 / 10$ & -40 & 40 & 24 & $3.66 *$ \\
\hline L sup. temporal & 6 & 21 & -55 & 5 & -12 & $5.35^{*}$ & & $38^{\dagger}$ & -51 & 11 & -9 & 2.76 \\
\hline R sup. temporal, STS & 7 & 22 & 53 & -19 & -1 & $4.47^{*}$ & 8 & 22 & 50 & -46 & 12 & $4.52 *$ \\
\hline $\mathrm{R}$ inf. parietal, supramarginal & & 40 & 61 & -38 & 24 & $3.72 *$ & 9 & 40 & 55 & -37 & 31 & $4.17^{*}$ \\
\hline L sup. temporal, STS & 8 & $42 / 22$ & -59 & -23 & 9 & $4.53 *$ & 10 & 22 & -59 & -25 & 7 & $3.64 *$ \\
\hline L sup. temporal & & 42 & -59 & -26 & 14 & $4.20 *$ & & 42 & -59 & -30 & 14 & $4.03 *$ \\
\hline L postcentral & & $2 / 40$ & -53 & -25 & 38 & $4.49 *$ & & 3 & -57 & -21 & 40 & $7.00 *$ \\
\hline L inf. parietal & & 40 & -65 & -24 & 29 & $3.63 *$ & & 40 & -65 & -24 & 29 & $4.60 *$ \\
\hline L sup. temporal/inf. parietal & 9 & $22 / 40$ & -57 & -44 & 15 & $4.77 *$ & & $22 / 40$ & -59 & -38 & 20 & $5.45^{*}$ \\
\hline $\mathrm{L}$ transverse temporal gyrus & 10 & 41 & -36 & -29 & 12 & $4.66 *$ & & $41^{\dagger}$ & -34 & -29 & 11 & 1.81 \\
\hline $\mathrm{R}$ inf. parietal/postcentral & 11 & $40 / 2$ & 46 & -31 & 46 & $4.37^{*}$ & 11 & 40 & 38 & -33 & 42 & $4.70 *$ \\
\hline $\mathrm{R}$ intraparietal, IPS & 12 & 40 & 38 & -42 & 44 & $4.05 *$ & 12 & $40 / 7$ & 44 & -46 & 56 & $4.14 *$ \\
\hline L intraparietal, IPS & 13 & 40 & -32 & -39 & 35 & $4.64 *$ & 13 & $7 / 40$ & -32 & -48 & 52 & $5.19 *$ \\
\hline R sup. parietal, precuneus & 14 & 7 & 14 & -74 & 42 & $4.81 *$ & & $7^{\dagger}$ & 16 & -65 & 51 & 2.73 \\
\hline L sup. parietal, precuneus & 15 & 7 & -12 & -59 & 35 & $4.40 *$ & & $7^{\dagger}$ & -12 & -61 & 53 & 2.83 \\
\hline
\end{tabular}

$\mathrm{BA}=$ Brodmann's area; $\mathrm{L}=$ left; $\mathrm{R}=$ right. $x, y, z$ express the position of the voxel with peak activation level $(p<.001$, uncorrected, extent threshold 10 voxels) within the cluster in millimeters relative to the anterior commissure (AC) in the stereotactic space (Talairach \& Tournoux, 1988).

$\dagger$ and italics indicate clusters that do not survive the threshold of $p<.001$, uncorrected, 10 voxels extent for the specific contrast. Here the highest $t$ value within the anatomically corresponding cluster is indicated. $x=$ lateral distance from the midline $(+$ right, - left $) ; y=$ anteroposterior distance from the AC (+ anterior, - posterior); $z=$ height relative to the AC line ( + above, - below). dPMC, vPMC $=$ dorsal, ventral premotor cortex; SMA $=$ supplementary motor area; STS $=$ superior temporal sulcus; IPS $=$ intraparietal sulcus; sup. $=$ superior; inf. $=$ inferior; mid. $=$ middle.

$* q<.05$, FDR corrected. 
in the right superior parietal (BA 7) and left superior temporal cortex (BA 22) (Table 3).

The direct comparison between pianists and controls while observing finger tapping movements without sound versus rest did not show any significant differences. Nevertheless, activation differences were more extensive and more significant for the contrast between observation of musical motor activity versus the resting condition compared to the contrast versus the nonmusical motor condition (Tables 1 and 3). We think that a "resting" hand constitutes a relatively low-level baseline condition compared to observation of finger tapping "movements." Accordingly, the direct comparison of observation of musical and nonmusical hand movements analyzes more specifically the aspect of the meaning and content of musical motor activity that is modulated by musical training in pianists.

\section{Recruitment of the Sensorimotor System by Auditory Input in Musicians}

Only a small subset of areas was activated stronger in musicians than controls when observing piano playing with sound compared to serial finger-thumb opposition movements with sound. For right-hand movements, the left inferior parietal cortex (BA 40/2), the dPMC bilaterally, and the left superior parietal cortex (BA 7) were activated stronger in musicians; for left-hand movements, only the right superior parietal cortex (BA 5/7) and left inferior parietal, somatosensory cortex (BA 1) were activated stronger in musicians. The small extent of those activation differences implies that in professional pianists, piano sound in association with serial finger-thumb opposition movements could cause activation within the fronto-parieto-temporal association network, therefore reducing activation differences between observation of piano-related and neutral movements with sound.

To further verify this aspect of activation of the sensorimotor system by auditory perception of piano sound in professional musicians, observation of serial fingerthumb opposition movements with and without sound was compared between both groups. We suppose those movements to have the same meaning and basic motor input for both groups and therefore activation differences are suggested to be due to perception and computing of piano sounds. Here significant signal increases were seen in pianists compared to controls in the left vPMC and bilaterally in the cerebellar hemispheres. Additionally, a trend to significance at $p<.01$ was detected in the inferior parietal cortex bilaterally, in the left parietal operculum, left superior parietal cortex, right prefrontal cortex, right superior temporal gyrus (BA 22), cingulate cortex (BA 24), and occipital cortex (BA 18, 19).

For all conditions, on-line monitoring of the EMG data during the test-run revealed no significant task- related activity within the flexor muscles of both forearms in any subject.

\section{DISCUSSION}

In this study, we show that observation and recognition of specific musical activity in professional pianists heavily relies on a multimodal fronto-parieto-temporal network including the inferior frontal gyrus (BA 44 and 45) and the ventral part of the lateral premotor cortex (vPMC) as well as the inferior parietal/intraparietal cortex and the temporal cortex within the STS and the adjacent temporal cortex (BA 21/22/42) bilaterally. Along with activation increases in dorsal fronto-parietal connections, this underlines the importance of this audiovisualsensorimotor system for musical skills that are developed by sustained training and learning.

Whereas most studies of action observation have examined object-related actions, our study represents the first investigation involving action observation of piano playing and finger-thumb opposition movements in professional pianists and musically naive controls. In line with our results, inferior frontal opercular activation differences at the ventral border to the precentral cortex in the context of motor observation are confirmed by recent fMRI data (Grezes, Armony, Rowe, \& Passingham, 2003; Hamzei et al., 2003). Despite substantial variability in the anatomy of human BA 44 and 45 (Amunts, Schleicher, et al., 1999), the stereotactic coordinates of our inferior frontal activation clusters are in agreement with previous data on activation patterns within the human inferior frontal cortex (Tomaiuolo et al., 1999). A meta-analysis of PET studies on various motor observation tasks as well as a recent fMRI study referring to mean coordinates of previous imaging studies on motor observation revealed comparable stereotactic localizations of activation within opercular parts of the inferior frontal gyrus (BA 44), vPMC, middle/superior temporal cortex, inferior parietal/intraparietal cortices, as well as dorsal fronto-parietal areas (Grezes, Armony, et al., 2003; Grezes \& Decety, 2001).

Within this network, the polysensory STS could integrate information about movement recognition with spatial information for the analysis of the meaning and goals of the observed behavior (Allison, Puce, \& McCarthy, 2000; Oram \& Perrett, 1996). This description could be further processed within the inferior parietal/ intraparietal cortex, which has been suggested to represent an interface, integrating somatosensory and visual information. STS and intraparietal areas have been described as heteromodal areas for cross-modal processing and integration of audiovisual information (Calvert, Hansen, Iversen, \& Brammer, 2001; Calvert, Campbell, \& Brammer, 2000). This analysis of the observed action can be relayed to neurons with motor properties in the inferior frontal cortex. Functionally, those neurons are 


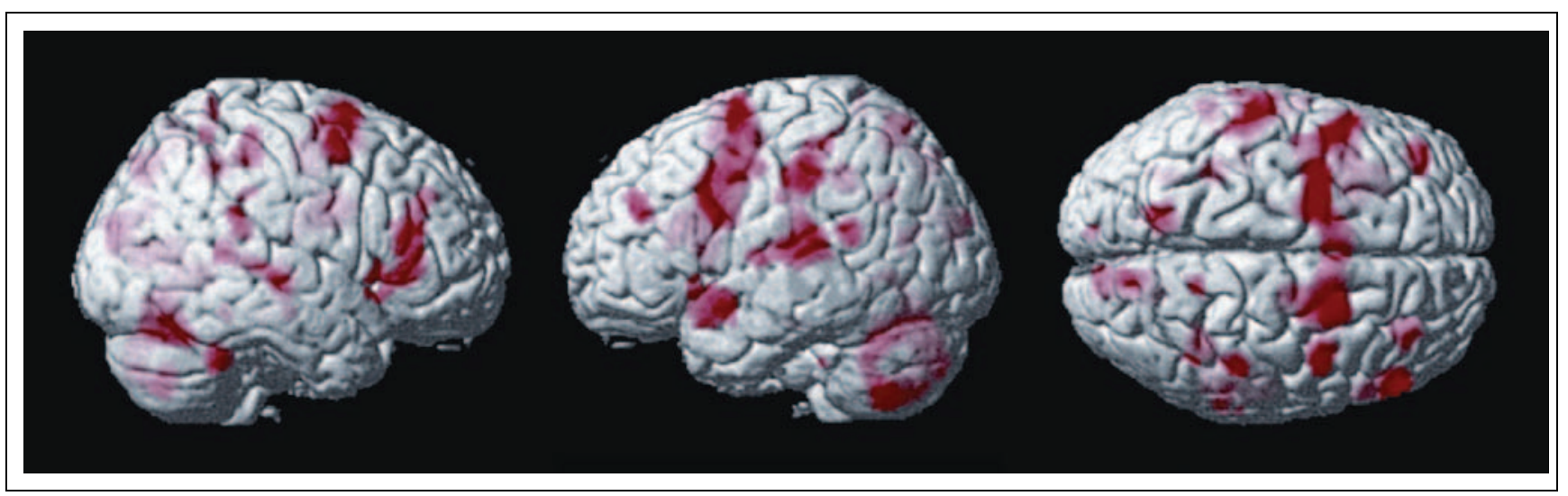

Figure 1. Areas showing significantly stronger activation in pianists than in controls during observation of right-hand piano playing movements without sound compared to observation of a resting hand. Areas of activation $(p<.001$, uncorrected) are projected onto a 3-D surface rendering of a stereotactically normalized T1-weighted magnetic resonance image. The depth of the activated voxels relative to the brain surface is coded in color intensity. The figure offers a view onto the right, left, and upper surface of the brain.

thought to describe an observed action in terms of its goal (Iacoboni, Woods, et al., 1999; Gallese \& Goldman, 1998; Jeannerod, Arbib, Rizzolatti, \& Sakata, 1995).

Beside this inferior fronto-parieto-temporal system, we also found a stronger involvement of dorsal frontoparietal connections in pianists compared to controls during observation of musical hand actions. The superior parieto-dorsal premotor circuit has been suggested to be responsible for motor control on the basis of somatosensory information (Rizzolatti, Luppino, \& Matelli, 1998). Superior parietal areas have been considered to integrate multimodal somatosensory information of the perceived movement (Binkofski, Buccino, et al., 1999; Grezes, Costes, \& Decety, 1999; Iacoboni, Woods, et al., 1999). (Pre)SMA has been supposed to control the selection and preparation of motor programs that are coded in the lateral parieto-frontal circuits. The prefrontal cortex provides inferior frontal areas with information about the meaning and goal of an observed action (Rizzolatti \& Luppino, 2001).

Bilateral activations of primary and secondary auditory areas were detected in pianists compared to controls when comparing observation of piano playing movements without sound to observation of a resting hand and of serial finger-thumb opposition movements without sound but not when opposing conditions with sound. This can be interpreted in the context of transmodal secondary auditory activation in musicians by visual perception of piano playing due to the development of cross-modal processes as a result of long-term musical training. Here, analogies can be established to the result of auditory activation by silent speech

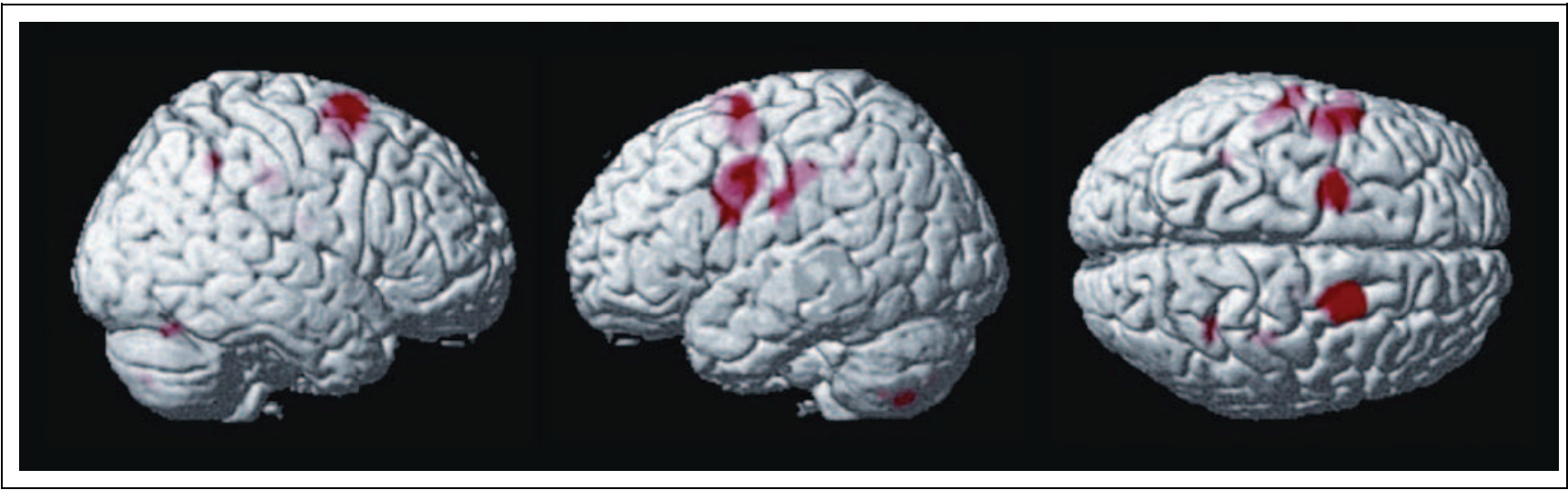

Figure 2. Areas showing significantly stronger activation in pianists than in controls during observation of right-hand piano playing movements without sound compared to observation of serial right-hand finger-thumb opposition movements without sound. Areas of activation $(p<.001$, uncorrected) are projected onto a 3-D surface rendering of a stereotactically normalized T1-weighted magnetic resonance image. The depth of the activated voxels relative to the brain surface is coded in color intensity. The figure offers a view onto the right, left, and upper surface of the brain. 
Table 2. Fronto-Parieto-Temporal Areas with Stronger Activations in Musicians than in Controls during Observation of Piano Playing with Sound Versus Observation of a Resting Hand

\begin{tabular}{|c|c|c|c|c|c|c|c|c|c|c|c|c|}
\hline \multirow{2}{*}{$\frac{\text { With Sound }}{\text { Area }}$} & \multicolumn{6}{|c|}{$R$ Piano $>$ Rest } & \multicolumn{6}{|c|}{ L Piano $>$ Rest } \\
\hline & Cluster & $B A$ & $x$ & $y$ & $z$ & Tlevel & Cluster & $B A$ & $x$ & $y$ & $z$ & T level \\
\hline $\mathrm{L}$ sup. frontal, $d P M C$ & 1 & 6 & -20 & -1 & 53 & $5.64 *$ & 1 & 6 & -20 & -1 & 55 & 5.47 \\
\hline $\mathrm{R}$ sup. frontal, $d P M C$ & & $\sigma^{\dagger}$ & 20 & 3 & 57 & 3.51 & 2 & 6 & 18 & 3 & 57 & 3.79 \\
\hline $\mathrm{L}$ inf. frontal, pars triangularis & & $45^{\dagger}$ & -53 & 14 & 1 & 2.00 & 3 & 45 & -53 & 18 & 5 & 4.89 \\
\hline $\mathrm{L}$ inf. frontal, pars opercularis & & $44^{\dagger}$ & -57 & 12 & 3 & 2.65 & & 44 & -51 & 12 & 5 & 4.24 \\
\hline $\mathrm{L}$ precentral, $v P M C$ & 2 & $6 / 44$ & -63 & 2 & 11 & $5.05^{*}$ & & 6 & -53 & 1 & 29 & 4.23 \\
\hline $\mathrm{R}$ inf. frontal, pars triangularis & & $45^{\dagger}$ & 53 & 31 & 6 & 2.83 & 4 & 45 & 50 & 25 & 4 & 3.95 \\
\hline L mid./inf. frontal & 3 & 9 & -40 & 27 & 26 & $5.00 *$ & & $9^{\dagger}$ & -59 & 15 & 27 & 2.72 \\
\hline $\mathrm{R}$ inf. parietal, supramarginal & 4 & 40 & 59 & -38 & 24 & $4.75^{*}$ & 5 & 40 & 67 & -33 & 29 & 3.93 \\
\hline $\mathrm{R}$ intraparietal, IPS & 5 & 40 & 46 & -46 & 56 & $4.45^{*}$ & & $40^{\dagger}$ & 40 & -50 & 58 & 2.18 \\
\hline $\mathrm{L}$ intraparietal, IPS & 6 & 40 & -48 & -36 & 50 & 3.93 & & $40^{\dagger}$ & -34 & -42 & 44 & 1.96 \\
\hline L postcentral, inf. parietal & 7 & $2 / 40$ & -65 & -20 & 25 & 4.06 & 6 & 1 & -57 & -25 & 40 & 4.47 \\
\hline R sup. parietal, precuneus & 8 & 7 & 14 & -74 & 44 & $5.08 *$ & & $7^{\dagger}$ & 22 & -55 & 58 & 2.22 \\
\hline L sup. parietal & 9 & 7 & -20 & -61 & 62 & 3.61 & & $7^{\dagger}$ & -28 & -48 & 59 & 2.92 \\
\hline
\end{tabular}

See notes to Table 1 for details.

$* q<.05$, FDR corrected.

perception as an example for cross-modal audiovisual processing (Calvert, Bullmore, et al., 1997).

Additionally, these auditory activations and also the greater activation differences in nonauditory cortical areas when contrasting observation of piano playing without sound to observation of a resting hand and of finger-thumb opposition movements without sound could partly be due to musical (auditory) imagination in our musicians. Aside from the temporal (auditory) cortices, the lateral frontal, mesial frontal (SMA), and parietal areas are activated during the auditory imagination of music (Halpern \& Zatorre, 1999; Zatorre, Halpern, Perry, Meyer, \& Evans, 1996). In contrast to previous data in nonmusicians showing a specialization of the right temporal cortex for musical imagery (Halpern \& Zatorre, 1999), our activations in musicians were lateralized to the left. As verbal processing during auditory imagery is thought to take place in the left superior temporal cortex (Halpern \& Zatorre, 1999), musicians might subconsciously attribute names of tones and intervals to the keys that are pressed by the observed hand. More likely, this shift from right hemispheric activation to left in musicians compared to nonmusicians can be hypothesized as some kind of automatic categorization, not necessarily verbal per se, that develops via musical training. The absence of auditory imagery could in part explain comparatively smaller activation differences when performing the same contrasts for observation of piano playing with sound.

Greater signal increases in pianists compared to controls within sensorimotor association areas due to the perception of piano tones synchronous to serial fingerthumb opposition movements suggest a transmodal recruitment of the sensorimotor system by auditory perception of musical information in musicians. This is further supported by small activation differences for both groups when comparing observation of meaningful piano playing movements with observation of serial finger-thumb opposition movements both with sound. It has previously been shown with EEG that after a certain time of musical practice already the isolated perception of sounds without visual movement perception leads to activation within the premotor, primary motor, parietal, and frontolateral areas that are also involved in actively playing a musical instrument (Bangert et al., 2001). Neurons within the inferior frontal cortex of the monkey have been shown to discharge in response to action-related sounds (Kohler et al., 2002). Learning-induced establishment of cross-modal links to an auditory input has been demonstrated with PET showing visual activation by isolated presentation of tones after a learning period with consistent pairing 
Table 3. Fronto-Parieto-Temporal Areas with Stronger Activations in Musicians than in Controls during Observation of Piano Playing without Sound Versus Observation of Finger-Thumb Opposition Movements without Sound

\begin{tabular}{|c|c|c|c|c|c|c|c|c|c|c|c|c|}
\hline \multirow{2}{*}{$\begin{array}{l}\text { Without Sound } \\
\text { Area }\end{array}$} & \multicolumn{6}{|c|}{$R$ Piano $>R F T$} & \multicolumn{6}{|c|}{$L$ Piano $>L F T$} \\
\hline & Cluster & $B A$ & $x$ & $y$ & $z$ & T level & Cluster & $B A$ & $x$ & $y$ & $z$ & T level \\
\hline $\mathrm{R}$ sup. frontal, $d P M C$ & 1 & 6 & 22 & 7 & 59 & $5.80 *$ & 1 & 6 & 22 & 9 & 62 & 4.27 \\
\hline L sup. frontal, $d P M C$ & 2 & 6 & -24 & 3 & 61 & $5.38 *$ & 2 & 6 & -22 & 3 & 55 & 4.43 \\
\hline L inf. frontal & 3 & 9 & -50 & 4 & 33 & $5.50 *$ & & $9^{\dagger}$ & -50 & 6 & 38 & 3.19 \\
\hline L inf. frontal, pars opercularis & & 44 & -59 & 7 & 20 & 4.24 & & $44^{\dagger}$ & -59 & 10 & 7 & 3.24 \\
\hline L mid. frontal, $v P M C$ & & 6 & -50 & -2 & 37 & $4.97^{*}$ & & $\sigma^{\dagger}$ & -51 & 4 & 35 & 2.84 \\
\hline $\mathrm{R}$ mid. frontal, $v P M C$ & & $\sigma^{\dagger}$ & 61 & 2 & 39 & 2.86 & 3 & 6 & 57 & 4 & 44 & 4.23 \\
\hline L sup. temporal & & $22^{\dagger}$ & -57 & -5 & 9 & 2.12 & 4 & 22 & -63 & -4 & 4 & 4.25 \\
\hline L sup. temporal & & $22^{\dagger}$ & -59 & -40 & 15 & 3.01 & 5 & 22 & -63 & -42 & 19 & 4.09 \\
\hline $\mathrm{R}$ postcentral & 4 & 2 & 38 & -23 & 38 & $4.47 *$ & & $40^{\dagger}$ & 40 & -34 & 51 & 2.85 \\
\hline $\mathrm{R}$ inf. parietal & & 40 & 38 & -29 & 35 & 3.78 & & & & & & \\
\hline L postcentral & 5 & 3 & -59 & -14 & 27 & 4.27 & 6 & $2 / 40$ & -59 & -23 & 45 & 4.25 \\
\hline L inf. parietal, operculum & & 43 & -63 & -13 & 19 & 3.69 & & $43^{\dagger}$ & -63 & -11 & 14 & 2.99 \\
\hline $\mathrm{R}$ inf. parietal, IPS & 6 & 40 & 34 & -48 & 43 & 4.18 & & $40^{\dagger}$ & 36 & -44 & 52 & 2.96 \\
\hline $\mathrm{L}$ inf. parietal, IPS & 7 & 40 & -36 & -41 & 39 & 3.81 & & $40^{\dagger}$ & -46 & -36 & 52 & 2.54 \\
\hline R sup. parietal & & $7^{\dagger}$ & 20 & -53 & 60 & 2.87 & 7 & 7 & 18 & -43 & 70 & 4.03 \\
\hline
\end{tabular}

FT = fingertapping; see notes to Table 1 for further details.

$* q<.05$, FDR corrected.

of both modalities (McIntosh, Cabeza, \& Lobaugh, 1998). The auditory perception of piano tones might therefore lead to a stronger recruitment of frontoparieto-temporal areas in pianists. This can explain reduced between-group activation differences in the motor observation network when comparing observation of piano playing and of serial finger-thumb opposition movements with sound.

Most of the fronto-parietal areas (apart from STS) involved in observation have also been shown to be activated to some extent during movement imagination (Binkofski, Amunts, et al., 2000; Grafton et al., 1996; Stephan et al., 1995; Decety, Perani, et al., 1994). Preparation of movement imitation (Krams, Rushworth, Deiber, Frackowiak, \& Passingham, 1998) and the intention to later imitate an action (Grezes, Costes, et al., 1999; Decety, Grezes, et al., 1997) have been suggested to modulate the recruitment of fronto-parietal areas during action observation. A higher level of attention associated with auditory imagery in conditions without sound or with motor imagery during perception of piano sound can in part account for greater activation of the fronto-parieto-temporal network in pianists during audiovisual perception of musical actions that have been practiced over many years. The action content and meaning that is modulated by regular practice influences activation of inferior frontal, inferior parietal as well as temporal motion areas. This is supported by previous data showing that especially observation of meaningful actions activates this cortical network (Decety, Grezes, et al., 1997). The perceived piano-related movements and sounds are aligned with an internal motor representation that has been established during many years of musical motor training and analyzed with regard to their goal and meaning.

It is interesting to note that in our study activation differences within the fronto-parieto-temporal network were generally more extensive and more significant for observation of right-hand piano playing. The only study that examined the effect of hand (right vs. left) on cerebral activation during motor observation demonstrated that during an observation-execution task with right hand motor responses, activation within the STS was greater for passive observation of a right hand 
compared to observation of left-hand movements (Iacoboni, Koski, et al., 2001). The authors hypothesized that during observation STS activity "reflects an implicit categorization of the moving hand as referred to the body of the observer." This contrasts a natural tendency to imitate movements in a mirror configuration (righthand imitation of left-hand action). Furthermore, the right-handedness of all subjects in our study and predominance of the right hand in most piano compositions may have contributed to stronger activation differences between pianists and controls for observation of righthand piano playing movements.

It is tempting to speculate that some aspects of the inferior fronto-parieto-temporal activation observed in the musicians reflect the operation of a "mirrormatching" system. Neurons with "mirror" properties (i.e., responding both to observation and execution of a specific action) are found in the monkeys' inferior frontal area $\mathrm{F} 5 \mathrm{C}$ (the homologue of human Broca's speech area) (Gallese, Fadiga, Fogassi, \& Rizzolatti, 1996; Rizzolatti, Fadiga, Gallese, \& Fogassi, 1996; di Pellegrino, Fadiga, Fogassi, Gallese, \& Rizzolatti, 1992), within inferior parietal cortex (area PF) (Fogassi, Gallese, Fadiga, \& Rizzolatti, 1998; Leinonen \& Nyman, 1979), as well as within the STS (Carey, Perrett, \& Oram, 1997; Perrett et al., 1989). F5 neurons have also been described to exhibit audiovisual properties responding to action-related sounds (Kohler et al., 2002). This audiovisual observation-execution matching system is supposed to be involved in translating perception to action by action recognition and understanding for preparation of adequate motor responses and movement imitation (Umilta et al., 2001; Gallese \& Goldman, 1998; Rizzolatti \& Arbib, 1998). Long-term musical motor learning and training by observation, listening, and imitation could support mirror-like neuronal computation.

The transmodal audiovisual network that we found stronger activated in pianists during passive observation of musical motor activity could functionally couple motor observation, auditory feedback, and imitation for learning of musical skills. A specialized system seems to develop during long-term piano training as an important element for the highly specific musical "intelligence" in professional musicians. This may constitute the neurobiological basis of imitative, observative, and auditory-based teaching elements that are successfully applied in music conservatories all over the world.

\section{METHODS}

\section{Subjects}

We investigated 12 professional pianists (6 women, 6 men, mean age $23.0 \pm 2.4$ years) and 12 musically naive control subjects ( 6 women, 6 men, mean age $25.0 \pm 1.2$ years). All subjects were strictly right handed according to the diagnostic criteria of the Edinburgh inventory (Oldfield, 1971). Musical professionalism was defined as having at least a completed conservatory degree in piano playing. Mean history of piano practice was $16.9 \pm 2.7$ years. Musically naive controls had to have never played a musical instrument and not to have received any musical teaching beyond normal school education. No subject had any history of neurological disease. All subjects were naive with regard to the goal of the study. All subjects gave written informed consent to the study in accordance with the guidelines from the Declaration of Helsinki. The study protocol was approved by the local Ethics Committee.

\section{Task}

The experimental task consisted in observation of different video sequences showing a real right or left hand performing piano playing movements or serial fingerthumb opposition movements with or without sound. The video image was projected onto a transparent screen using a video beamer. The scanning room was completely darkened. Subjects viewed the screen by means of a mirror that was centered above their eyes. The video scenes were $<10^{\circ}$ wide and $<7.5^{\circ}$ high as seen by the subjects with hand movements being centered to a range of $<3.5^{\circ}$ in width. The sound was transmitted to the subjects via pneumatic earphones (EAR-Link 3a Cabot Safety, Indianapolis, IN, USA). Subjects were instructed only to watch the hand and to keep their forearm, hand, or fingers completely relaxed. Both arms were kept supine, loosely enclosing a foam pad in order to achieve a position that least resembles a natural piano position. Five different conditions were presented each for right- and left-hand movements respectively:

First, sequences showed a right or left hand from a bird's eye view continuously performing silent monophonic piano playing movements pressing one key at a time with a finger on a typical piano keyboard. Movements were performed at a speed of $1 \mathrm{~Hz}$ within an interval of 10 tones, thereby avoiding extensive hand movements. Finger movements were nonsequential, alternatingly involving all fingers of the hand and "randomly" distributed over all keys within the interval (Figure 3A).

In a second condition, video sequences were shown like in the first condition with the difference that the corresponding piano sound was transmitted synchronous to the keypresses. Corresponding to the random distribution of finger movements over the keys, this sound was no real melody or scale but a random sequence of single tones. We chose this kind of random and nonserial finger movements corresponding to nonmelodic random sequences of piano tones because we were interested in the "basic" aspects of motor observation and sound perception. This avoids confounding the results with "higher" aspects of musical perception and musicality that cannot be compared between professional musicians and musically naive controls. 


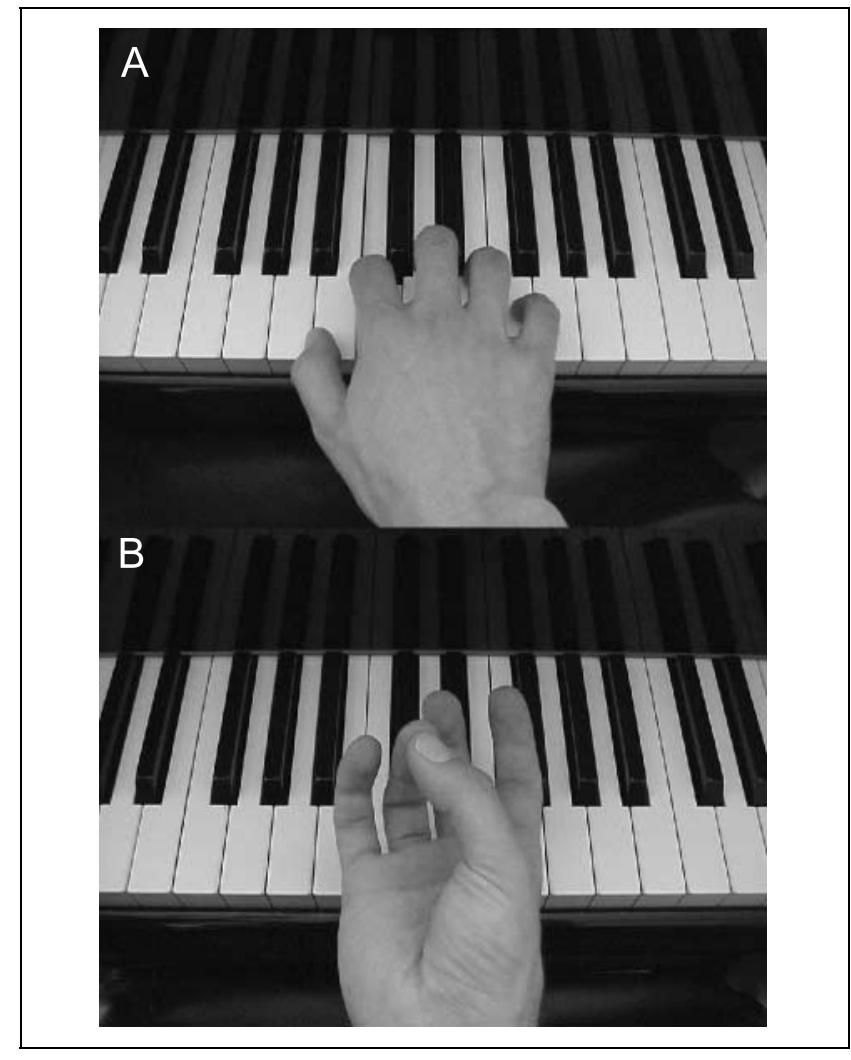

Figure 3. Examples of the activation paradigm. The figure shows a right hand performing (A) "meaningful" piano playing movements on a keyboard and (B) "neutral" sequential finger to thumb opposition movements supine in front of the keyboard. Both conditions were presented with and without synchronous piano sound.

During the third condition, sequential finger to thumb opposition movements with the right or left hand supine in front of the piano keyboard had to be observed (Figure 3B). These movements serially involved finger to thumb oppositions of the index, middle, ring, and little finger in bidirectional order and were equally performed at a frequency of $1 \mathrm{~Hz}$ without any sound.

The same sequential finger-thumb opposition movements were presented to the subjects as above in a fourth condition where piano sound, representing random tonal sequences as in the piano conditions, was transmitted synchronous to the finger movements. Piano tones appeared at a frequency of $1 \mathrm{~Hz}$ simultaneous to the time of contact between finger and thumb.

A right or left hand resting prone on the piano keyboard was displayed as control condition.

\section{Data Acquisition}

fMRI measurements were performed on a $1.5 \mathrm{~T}$ Philips Gyroscan NT scanner (Hamburg, Germany) upgraded to Gyroscan Intera and equipped with a circular polarized birdcage head coil. A forehead restraining strip and various foam pads served for head fixation. The functional scans were acquired using a high-speed gradient- weighted EPI sequence (TR $3 \mathrm{sec}$, TE $50 \mathrm{msec}$, flip angle $90^{\circ}$, FOV $230 \mathrm{~mm}$, matrix $64 \times 64$ resulting in an in plane resolution of $3.59 \mathrm{~mm}$ ). A volume of 30 continuous axial slices parallel to the AC-PC line with a single slice thickness of $5 \mathrm{~mm}$ covering the whole brain was recorded during each scan. Additionally, a whole-brain 3-D T1-weighted image was acquired for each subject for high-resolution anatomical reference. The audio system sound pressure level was optimized before each single scanning session so that subjects could clearly hear the sound even during fMRI scanning.

We performed four block-design functional runs per subject at 100 scans each with a single run duration of $300 \mathrm{sec}$. Two fixed experimental runs were designed for each hand separately involving only observation of rightor left-hand movements, respectively. Each run was grouped into 10 epochs of 10 whole-brain EPI scans $(30 \mathrm{sec})$. For the first run per hand, the five conditions were alternated over epochs and repeated twice during the run. The epoch order of the second run per hand was derived from the first by mirroring the epoch order before and after the fifth epoch. The same four experimental runs were presented for all subjects in pseudorandomized order, thereby always alternating left- and right-hand runs.

For controlling of eventual finger or hand movements during motor observation, surface EMG electrodes were attached over the forearm flexor muscles of both arms. An additional experimental run without fMRI scanning was performed at the end of the session for two-channel surface EMG recordings. Subjects were naive about the goal of this extra run.

The EMG amplifier as well as the video recorder and the fMRI scanner were linked to a Lab-View based electrophysiological monitoring system which allowed the precise on-line registration of the EMG signal and the fMRI timing parameters along with the experimental paradigm (piano sound).

\section{Data Analysis}

Data were analyzed using SPM99 software (Wellcome Department of Cognitive Neurology, www.fil.ion.ucl. ac.uk/spm) based on the general linear model (Friston et al., 1995). Calculations were performed on PCs running LINUX and Matlab 5.3 (Mathworks, Sherborn, MA). The first two scans of each run were omitted to allow equilibration of saturation effects resulting in a total of 98 scans per run. Preprocessing of the data took several steps: Images of each subject were realigned to the first image of the series, stereotactically normalized into a standard space (Montreal Neurological Institute, MNI) approximating that of Talairach and Tournoux (1988) and smoothed with a gaussian filter of $12 \times 12 \times 12 \mathrm{~mm}^{3}$.

A second-level random-effects approach was applied for statistical analysis. On the first level, the four functional runs were entered into an individual design matrix 
for each subject. Here four different conditions per run, observation of right-hand piano playing or finger-thumb opposition movements each with or without sound and accordingly observation of left-hand piano playing or finger-thumb opposition movements each with or without sound, were defined explicitly with the control condition modeled implicitly. Data were convolved with a canonical hemodynamic response function. A highpass filter was applied for filtering low-frequency noise (range of cutoffs: 240-264 sec). Four different contrasts were defined comparing observation of piano playing movements (Piano) without or with piano sound (Silent/ Sound) to observation of sequential finger-thumb opposition movements (Sequence) and to observation of a resting hand (Rest) separately for right- and left-hand movements, respectively. Additionally, to detect if perception of piano sound in association with serial fingerthumb opposition movements leads to an increase in sensorimotor activation, we compared observation of finger-thumb opposition movements with sound (both right and left hand together) to observation of fingerthumb opposition movements without sound. This resulted in a total of nine contrast images per subject:

1. Right Piano Silent-Right Rest

2. Right Piano Sound-Right Rest

3. Right Piano Silent-Right Sequence Silent

4. Right Piano Sound-Right Sequence Sound

5. Left Piano Silent-Left Rest

6. Left Piano Sound-Left Rest

7. Left Piano Silent-Left Sequence Silent

8. Left Piano Sound-Left Sequence Sound

9. Right + Left Sequence Sound-Right + Left Sequence Silent

The corresponding contrast images were entered into a second level (random effects) analysis for group comparison. A two-sample $t$ test was applied for calculating activation differences between the group of professional pianists and the group of musical laymen. Based on the results of previous functional imaging studies on motor observation and our a priori hypotheses, a statistical threshold of $p<.001$, uncorrected (10 voxels extent threshold), was considered to show significant activation. To further corroborate the findings a threshold of $p<.05$ FDR corrected was applied to the data (Genovese, Lazar, \& Nichols, 2002). For localization within the stereotactic space of Talairach (Talairach \& Tournoux, 1988), the coordinates of activated voxels were transformed from MNI to Talairach coordinates using a routine proposed by M. Brett (www.mrc-cbu. cam.ac.uk/Imaging/mnispace.html).

\section{Acknowledgments}

This work was supported by the SFB 462 "Sensomotorik," Deutsche Forschungsgemeinschaft Bonn and the "Gerhard und Irmgard Schulz Fond," Neurologische Klinik, Klinikum
Rechts der Isar. We thank all subjects, especially the professional pianists, for participating in the study. Special thanks to Martina Bauer ("Hochschule für Musik und Theater," Munich) and Dr. Thomas Hitzlberger ("Richard Strauss Konservatorium," Munich) for their support in recruiting the pianists.

Reprint requests should be sent to Bernhard Haslinger, Neurologische Klinik und Poliklinik, TU-München Möhlstraße 28 D-81675 Munich, or via e-mail: b.haslinger@lrz.tumuenchen.de.

The data reported in this experiment have been deposited in the fMRI Data Center (www.fmridc.org). The accession number is $2-2004-1177 \mathrm{X}$.

\section{REFERENCES}

Allison, T., Puce, A., \& McCarthy, G. (2000). Social perception from visual cues: Role of the STS region. Trends in Cognitive Sciences, 4, 267-278.

Amunts, K., Schlaug, G., Jäncke, L., Steinmetz, H., Schleicher, A., Dabringhaus, A., \& Zilles, K. (1997). Motor cortex and hand motor skills: Structural compliance in the human brain. Human Brain Mapping, 5, 206-215.

Amunts, K., Schleicher, A., Burgel, U., Mohlberg, H., Uylings, H. B., \& Zilles, K. (1999). Broca's region revisited: Cytoarchitecture and intersubject variability. Journal of Comparative Neurology, 412, 319-341.

Bangert, M., Haeusler, U., \& Altenmuller, E. (2001). On practice: How the brain connects piano keys and piano sounds. Annals of the New York Academy of Sciences, 930, 425-428.

Binkofski, F., Amunts, K., Stephan, K. M., Posse, S., Schormann, T., Freund, H. J., Zilles, K., \& Seitz, R. J. (2000). Broca's region subserves imagery of motion: A combined cytoarchitectonic and fMRI study. Human Brain Mapping, 11, 273-285.

Binkofski, F., Buccino, G., Posse, S., Seitz, R. J., Rizzolatti, G., \& Freund, H. (1999). A fronto-parietal circuit for object manipulation in man: Evidence from an fMRI-study. European Journal of Neuroscience, 11, 3276-3286.

Bonda, E., Petrides, M., Ostry, D., \& Evans, A. (1996). Specific involvement of human parietal systems and the amygdala in the perception of biological motion. Journal of Neuroscience, 16, 3737-3744.

Buccino, G., Binkofski, F., Fink, G. R., Fadiga, L., Fogassi, L., Gallese, V., Seitz, R. J., Zilles, K., Rizzolatti, G., \& Freund, H. J. (2001). Action observation activates premotor and parietal areas in a somatotopic manner: An fMRI study. European Journal of Neuroscience, 13, 400-404.

Calvert, G. A., Bullmore, E. T., Brammer, M. J., Campbell, R., Williams, S. C., McGuire, P. K., Woodruff, P. W., Iversen, S. D., \& David, A. S. (1997). Activation of auditory cortex during silent lipreading. Science, 276, 593-596.

Calvert, G. A., Campbell, R., \& Brammer, M. J. (2000). Evidence from functional magnetic resonance imaging of crossmodal binding in the human heteromodal cortex. Current Biology, 10, 649-657.

Calvert, G. A., Hansen, P. C., Iversen, S. D., \& Brammer, M. J. (2001). Detection of audio-visual integration sites in humans by application of electrophysiological criteria to the BOLD effect. Neuroimage, 14, 427-438.

Carey, D. P., Perrett, D. I., \& Oram, M. W. (1997). Recognizing, understanding and reproducing actions. In J. Grafman (Ed.), Action and cognition: Handbook of neuropsychology (vol. 11, pp. 111-130). Amsterdam: Elsevier. 
Decety, J., Grezes, J., Costes, N., Perani, D., Jeannerod, M., Procyk, E., Grassi, F., \& Fazio, F. (1997). Brain activity during observation of actions. Influence of action content and subject's strategy. Brain, 120, 1763-1777.

Decety, J., Perani, D., Jeannerod, M., Bettinardi, V., Tadary, B., Woods, R., Mazziotta, J. C., \& Fazio, F. (1994). Mapping motor representations with positron emission tomography. Nature, 371, 600-602.

di Pellegrino, G., Fadiga, L., Fogassi, L., Gallese, V., \& Rizzolatti, G. (1992). Understanding motor events: A neurophysiological study. Experimental Brain Research, 91, 176-180.

Elbert, T., Pantev, C., Wienbruch, C., Rockstroh, B., \& Taub, E. (1995). Increased cortical representation of the fingers of the left hand in string players. Science, 270, 305-307.

Fadiga, L., Fogassi, L., Pavesi, G., \& Rizzolatti, G. (1995). Motor facilitation during action observation: A magnetic stimulation study. Journal of Neurophysiology, 73, 2608-2611.

Fogassi, L., Gallese, V., Fadiga, L., \& Rizzolatti, G. (1998). Neurons responding to the sight of goal-directed hand/arm actions in the parietal area PF (7b) of the macaque monkey. Society of Neuroscience Abstracts, 24, 654.

Friston, K. J., Holmes, A. P., Worsley, K. J., Poline, J.-B., Frith, C. D., \& Frackowiak, R. S. J. (1995). Statistical parametric maps in functional imaging: A general linear approach. Human Brain Mapping, 2, 189-210.

Gallese, V., Fadiga, L., Fogassi, L., \& Rizzolatti, G. (1996). Action recognition in the premotor cortex. Brain, 119, 593-609.

Gallese, V., \& Goldman, A. (1998). Mirror neurons and the simulation theory of mind-reading. Trends in Cognitive Sciences, 2, 493-501.

Genovese, C. R., Lazar, N. A., \& Nichols, T. (2002). Thresholding of statistical maps in functional neuroimaging using the false discovery rate. Neuroimage, $15,870-878$.

Grafton, S. T., Arbib, M. A., Fadiga, L., \& Rizzolatti, G. (1996). Localization of grasp representations in humans by positron emission tomography: 2. Observation compared with imagination. Experimental Brain Research, 112, 103-111.

Grezes, J., Armony, J. L., Rowe, J., \& Passingham, R. E. (2003). Activations related to "mirror" and "canonical" neurones in the human brain: An fMRI study. Neuroimage, 18, 928-937.

Grezes, J., Costes, N., \& Decety, J. (1999). The effects of learning and intention on the neural network involved in the perception of meaningless actions. Brain, 122, 1875-1887.

Grezes, J., \& Decety, J. (2001). Functional anatomy of execution, mental simulation, observation, and verb generation of actions: A meta-analysis. Human Brain Mapping, 12, 1-19.

Halpern, A. R., \& Zatorre, R. J. (1999). When that tune runs through your head: A PET investigation of auditory imagery for familiar melodies. Cerebral Cortex, 9, 697-704.

Hamzei, F., Rijntjes, M., Dettmers, C., Glauche, V., Weiller, C., \& Buchel, C. (2003). The human action recognition system and its relationship to Broca's area: An fMRI study. Neuroimage, 19, 637-644.

Hari, R., Forss, N., Avikainen, S., Kirveskari, E., Salenius, S., \& Rizzolatti, G. (1998). Activation of human primary motor cortex during action observation: A neuromagnetic study. Proceedings of the National Academy of Sciences, U.S.A., 95, 15061-15065.

Iacoboni, M., Koski, L. M., Brass, M., Bekkering, H., Woods, R. P., Dubeau, M. C., Mazziotta, J. C., \& Rizzolatti, G. (2001). Reafferent copies of imitated actions in the right superior temporal cortex. Proceedings of the National Academy of Sciences, U.S.A., 98, 13995-13999.
Iacoboni, M., Woods, R. P., Brass, M., Bekkering, H., Mazziotta, J. C., \& Rizzolatti, G. (1999). Cortical mechanisms of human imitation. Science, 286, 2526-2528.

Jeannerod, M., Arbib, M. A., Rizzolatti, G., \& Sakata, H. (1995). Grasping objects: The cortical mechanism of visuomotor transformation. Trends in Neurosciences, 18 , 314-320.

Kohler, E., Keysers, C., Umilta, M. A., Fogassi, L., Gallese, V., \& Rizzolatti, G. (2002). Hearing sounds, understanding actions: Action representations in mirror neurons. Science, 297, 846-848.

Krams, M., Rushworth, M. F., Deiber, M. P., Frackowiak, R. S., \& Passingham, R. E. (1998). The preparation, execution and suppression of copied movements in the human brain. Experimental Brain Research, 120, 386-398.

Leinonen, L., \& Nyman, G. (1979). II. Functional properties of cells in anterolateral part of area 7 associative face area of awake monkeys. Experimental Brain Research, 34, 321-333.

McIntosh, A. R., Cabeza, R. E., \& Lobaugh, N. J. (1998). Analysis of neural interactions explains the activation of occipital cortex by an auditory stimulus. Journal of Neurophysiology, 80, 2790-2796.

Nishitani, N., \& Hari, R. (2000). Temporal dynamics of cortical representation for action. Proceedings of the National Academy of Sciences, U.S.A., 97, 913-918.

Oldfield, R. C. (1971). The assessment and analysis of handedness: The Edinburgh inventory. Neuropsychologia, 9, 97-113.

Oram, M. W., \& Perrett, D. I. (1996). Integration of form and motion in the anterior superior temporal polysensory area (STPa) of the macaque monkey. Journal of Neurophysiology, 76, 109-129.

Pantev, C., Oostenveld, R., Engelien, A., Ross, B., Roberts, L. E., \& Hoke, M. (1998). Increased auditory cortical representation in musicians. Nature, 392, 811-814.

Perani, D., Fazio, F., Borghese, N. A., Tettamanti, M., Ferrari, S., Decety, J., \& Gilardi, M. C. (2001). Different brain correlates for watching real and virtual hand actions. Neuroimage, 14, 749-758.

Perrett, D. I., Harries, M. H., Bevan, R., Thomas, S., Benson, P. J., Mistlin, A. J., Chitty, A. J., Hietanen, J. K., \& Ortega, J. E. (1989). Frameworks of analysis for the neural representation of animate objects and actions. Journal of Experimental Biology, 146, 87-113.

Rizzolatti, G., \& Arbib, M. A. (1998). Language within our grasp. Trends in Neurosciences, 21, 188-194.

Rizzolatti, G., Fadiga, L., Gallese, V., \& Fogassi, L. (1996). Premotor cortex and the recognition of motor actions. Brain Research, Cognitive Brain Research, 3, 131-141.

Rizzolatti, G., Fadiga, L., Matelli, M., Bettinardi, V., Paulesu, E., Perani, D., \& Fazio, F. (1996). Localization of grasp representations in humans by PET: 1 . Observation versus execution. Experimental Brain Research, 111, $246-252$.

Rizzolatti, G., \& Luppino, G. (2001). The cortical motor system. Neuron, 31, 889-901.

Rizzolatti, G., Luppino, G., \& Matelli, M. (1998). The organization of the cortical motor system: New concepts. Electroencephalography and Clinical Neurophysiology, 106, 283-296.

Schlaug, G., Jancke, L., Huang, Y., Staiger, J. F., \& Steinmetz, H. (1995). Increased corpus callosum size in musicians. Neuropsychologia, 33, 1047-1055.

Schlaug, G., Jancke, L., Huang, Y., \& Steinmetz, H. (1995). In vivo evidence of structural brain asymmetry in musicians. Science, 267, 699-701. 
Schneider, P., Scherg, M., Dosch, H. G., Specht, H. J., Gutschalk, A., \& Rupp, A. (2002). Morphology of Heschl's gyrus reflects enhanced activation in the auditory cortex of musicians. Nature Neuroscience, 5, 688-694.

Sergent, J., Zuck, E., Terriah, S., \& MacDonald, B. (1992). Distributed neural network underlying musical sight-reading and keyboard performance. Science, 257, 106-109.

Sluming, V., Barrick, T., Howard, M., Cezayirli, E., Mayes, A., \& Roberts, N. (2002). Voxel-based morphometry reveals increased gray matter density in Broca's area in male symphony orchestra musicians. Neuroimage, 17, 1613-1622.

Stephan, K. M., Fink, G. R., Passingham, R. E., Silbersweig, D., Ceballos-Baumann, A. O., Frith, C. D., \& Frackowiak, R. S. (1995). Functional anatomy of the mental representation of upper extremity movements in healthy subjects.

Journal of Neurophysiology, 73, 373-386.

Talairach, J., \& Tournoux, P. A. (1988). Co-planar stereotactic atlas of the buman brain. Stuttgart: Thieme.
Tomaiuolo, F., MacDonald, J. D., Caramanos, Z., Posner, G., Chiavaras, M., Evans, A. C., \& Petrides, M. (1999).

Morphology, morphometry and probability mapping of the pars opercularis of the inferior frontal gyrus: An in vivo MRI analysis. European Journal of Neuroscience, 11, 3033-3046.

Umilta, M. A., Kohler, E., Gallese, V., Fogassi, L., Fadiga, L., Keysers, C., \& Rizzolatti, G. (2001). I know what you are doing. A neurophysiological study. Neuron, 31, $155-165$.

Zatorre, R. J., Halpern, A. R., Perry, D. W., Meyer, E., \& Evans, A. C. (1996). Hearing in the mind's ear: A PET investigation of musical imagery and perception. Journal of Cognitive Neuroscience, 8, 29-46.

Zatorre, R. J., Perry, D. W., Beckett, C. A., Westbury, C. F., \& Evans, A. C. (1998). Functional anatomy of musical processing in listeners with absolute pitch and relative pitch. Proceedings of the National Academy of Sciences, U.S.A., 95, 3172-3177. 